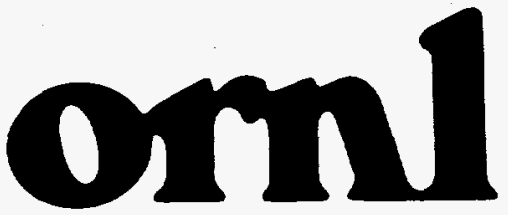

OAK RIDGE

NATIONAL

LABORATORY

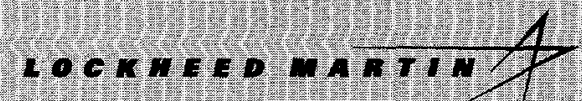

(1)
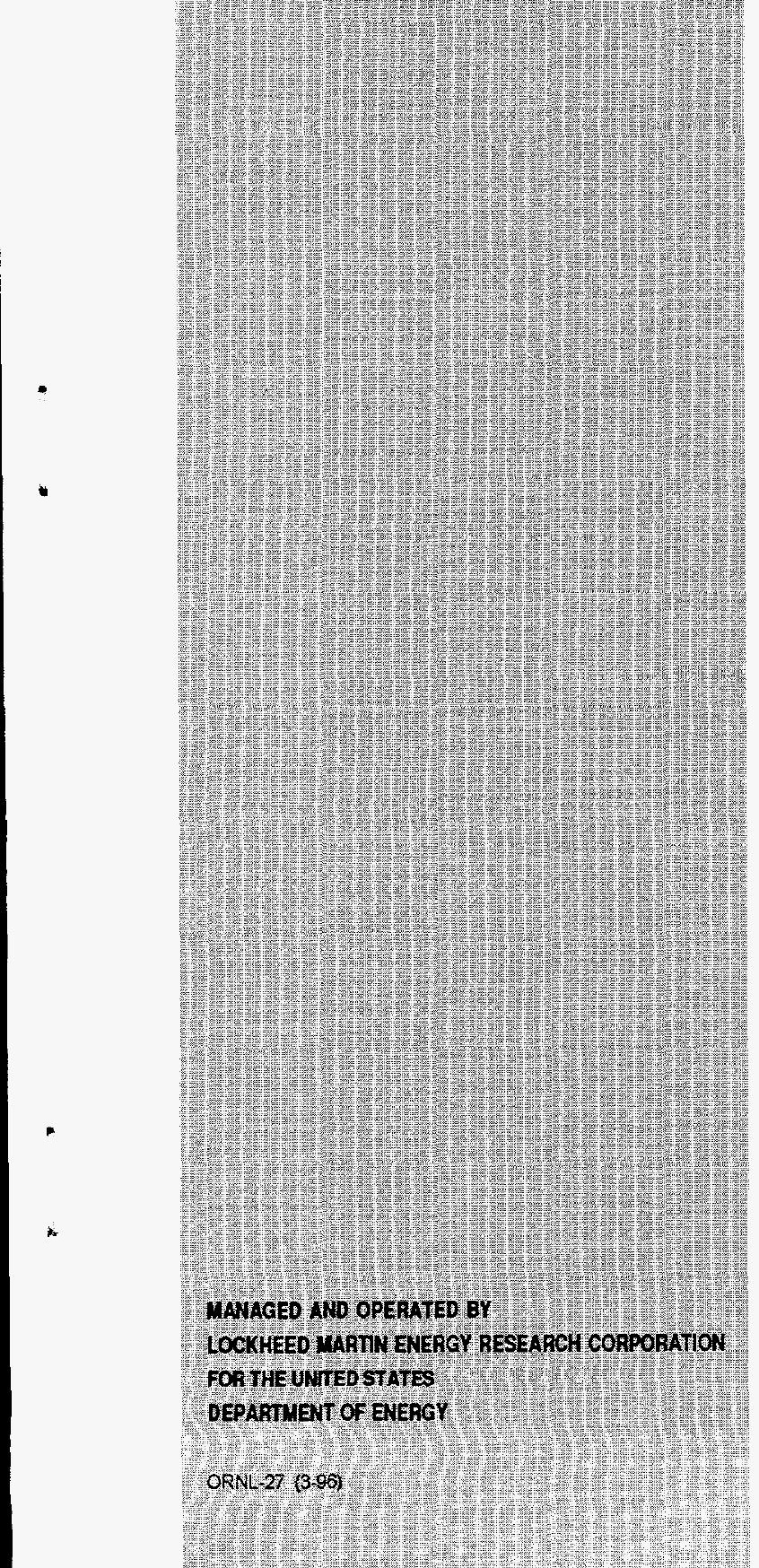

MAMGED IND OPERATED BY LOCKHEED WARTI ENERGY RESEAPCH CORPORATION FORTHEUNTEB STATES DEPARTMENT OF ENERGY 
This report has been reproduced directly from the best available copy.

Available to DOE and DOE contractors from the Office of Scientific and Technical

Information, P.O.Box 62, Oak Ridge, TN 37831; prices available from (423) 576-8401, FTS 626-8401.

Available to the public from the National Technical Information Service, U.S. Department

of Commerce, 5285 Port Royal Rd., Springfield, VA 22161

This report was prepared a an account of work sponsored by an agency of the United States Government. Neither the United States Government nor any agency thereof, nor any of their employees, makes any warranty, express or implied, or assumes any legal liability or responsibility for the accuracy, completeness, or usefulness of any information, apparatus, product, or process disclosed, or represents that its use would not infringe privately owned rights. Reference herein to any specific commercial product, process, or service by trade name, trademark, manufacturer, or otherwise, does not necessarily constitute or imply its endorsement, recommendation, or favoring by the United States Government or any agency thereof. The view and opinions of authors expressed herein do not necessarily state or reflect those of the UnitedStatesGovernment or any agency thereof. 


\section{DISCLAIMER}

Portions of this document may be illegible electronic image products. Images are produced from the best available original document. 
Computer Science and Mathematics Division

Distributed Intelligent Systems Section

\title{
A Comparison of Three Redundancy Resolution Methods
}

\author{
M. A. Unseren \\ Oak Ridge National Laboratory \\ P.O. Box 2008, Bldg. 6025 \\ Oak Ridge, TN 37831-6364 \\ myu@ornl.gov \\ and \\ D. B. Reister \\ Oak Ridge National Laboratory \\ P.O. Box 2008, Bldg. 6010 \\ Oak Ridge, TN 37831-6355 \\ dbr@ornl.gov
}

Date Published: October 1997

Research was supported by the Engineering Research

Program of the Office of Basic Energy Sciences, U.S.

Department of Energy

\author{
Prepared by \\ Oak Ridge National Laboratory \\ Oak Ridge, Tennessee 37831 \\ managed by \\ Lockheed Martin Energy Research Corporation \\ for the \\ U.S. DEPARTMENT OF ENERGY \\ under Contract No. DE-AC05-96OR22464
}





\section{Contents}

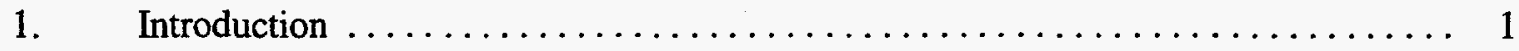

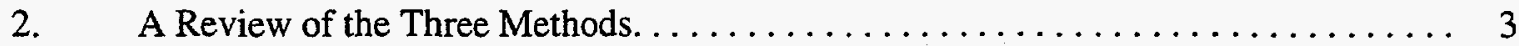

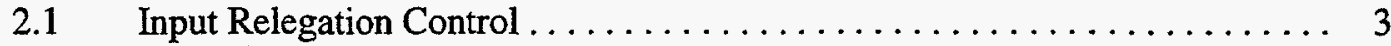

$2.2 \quad$ Belmans-Culioli Method ......................... 4

$2.3 \quad$ Full Space Parameterization Method. .................... 5

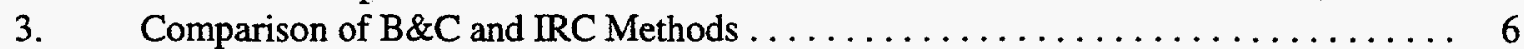

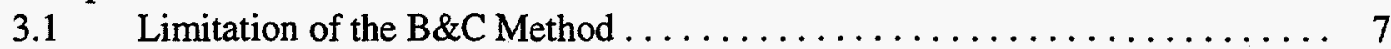

3.2 Linear Relationship Between $\in$ and $\mu \ldots \ldots \ldots \ldots \ldots \ldots \ldots . \ldots \ldots$

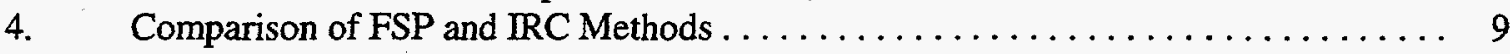

$4.1 \quad$ Limitation of the FSP Method $\ldots \ldots \ldots \ldots \ldots \ldots \ldots \ldots \ldots \ldots \ldots$

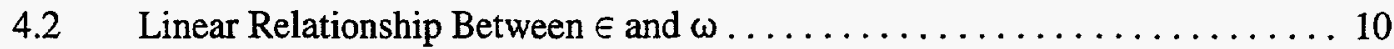

5. Unification of B\&C and FSP Null Space Parameterizations and Solution Methods ... 11

6. Discussion and Conclusion. .............................. 12

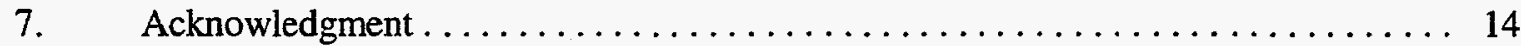

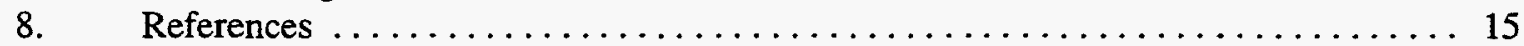

9. Appendix A: Comparison of IRC to Constraint Free Representations of B\&C and FSP Methods. 


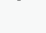




\begin{abstract}
The report performs an analytical comparison of three redundancy resolution methods developed by researchers at the Oak Ridge National Laboratory that obtain solutions for the joint velocities for a redundant manipulator based on its kinematic velocity model with an $(m \times n)$ Jacobian matrix of full rank $m(<n)$ : (i) the Input Relegation Control (IRC) Method $[1,2]$; the Belmans and Culioli (B\&C) method $[3,4]$; and an extension of the $B \& C$ method termed Full Space Parameterization (FSP) [5]. Several new insights into the B\&C joint velocity solution are obtained: (i) only the self motion component $\$$ of the solution is a function of the $p=(n-m+1)$ scalar variables $\left\{\mu_{1}, \mu_{2}, \ldots, \mu_{p}\right\}$ subjected to the constraint $\sum_{k=1}^{p} \mu_{k}=1$ introduced in [3,4]; (ii) these variables along with the constraint, in fact parameterize the null space of the Jacobian; and (iii) self motion inducing joint velocities cannot be obtained by the $\mathrm{B} \& \mathrm{C}$ solution when the end effector is stationary. Indeed, all joint velocities in the B\&C solution are identically equal to zero in this configuration. The IRC solution, which parameterizes the null space using a minimal number $(n-m)$ of variables, can induce joint self motion when the end effector is stationary. A new constraint free representation of the $B \& C$ joint velocity solution is derived and it is shown that it too has the limitation described in (iii). The FSP method yields a joint velocity solution which induces joint self motion but does not induce end effector motion. The FSP solution is a linear function of $p$ variables $\left\{\omega_{1}, \omega_{2}, \ldots, \omega_{p}\right\}$ which parameterize the null space provided that they satisfy $\sum_{k=1}^{p} \omega_{k}=0$. A new insight reveals that all joint velocities in the FSP solution are identically equal to zero when the end effector is stationary, and a constraint free representation of the FSP solution derived here possesses the same limitation. The FSP method cannot induce joint self motion velocities in this configuration. Finally, it is demonstrated that if the null space is parameterized using the quantities $\left\{\mu_{1}, \mu_{2}, \ldots, \mu_{p}\right\}$ subjected to the constraint $\sum_{k=1}^{p} \mu_{k}=1$, then introducing the quantities $\left\{\omega_{1}, \omega_{2}, \ldots, \omega_{p}\right\}$ subjected to the constraint $\sum_{k=1}^{p} \omega_{k}=0$ provides no new information.
\end{abstract}

\footnotetext{
$\S$ Joint self motion or self motion is defined to be motion of the joints that does not affect the Cartesian space motion of the manipulator's end effector.
} 


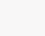




\section{Introduction}

There is a proliferation of methods $[6,7]$ for resolving the kinematic redundancy of a fixed base, serial link manipulator based on its kinematic velocity model:

$$
\dot{x}=J \dot{q}
$$

where $\dot{q}$ is the $(n \times 1)$ vector of joint velocities and $J=J(q)$ is the $(m \times n)$ Jacobian matrix which is assumed to possess full rank $m(<n)$. It is assumed that a trajectory for the end effector has been specified, thus the $(m \times 1)$ vector of Cartesian velocities $\dot{x}$ is a known quantity in eq. (1).

Three such methods for determining solutions for joint velocities based on eq. (1) have been developed by researchers at the Oak Ridge National Laboratory (ORNL), namely: (i) the Input Relegation Control (IRC) Method [1, 2]; (ii) the Belmans and Culioli (B\&C) method $[3,4]$; and (iii) an extension of the $B \& C$ method termed Full Space Parameterization (FSP) [5]. Naturally, there should be some connection among the methods, but to date there has been no investigation of a possible connection among them. Therefore, the purpose of this paper is to perform an analytical comparison of the three methods with added focus on identifying limitations and/or deficiencies associated with them.

As will be reviewed, a key feature of the IRC method is that it describes all possible solutions to eq. (1) as linear combinations of the exact range space of $J^{T}$ and the null space of $J$ (here superscript $T$ denotes a transpose). With IRC, the known vector $\dot{x}$ parameterizes the former subspace and the latter is parameterized using a minimal number of independent variables. Since the exact range space of $J^{T}$ and the null space (of $J$ ) span the $n$-dimensional articular space, all other solution methods can be expressed in terms of this basis. After reviewing the latter two methods, the comparison commences by expressing the solution for the joint velocities obtained by the $B \& C$ method in terms of the IRC basis which separates it into its end effector motion inducing and self motion inducing components. This leads to several new insightful observations about the B\&C solution: (i) only the self motion component is a function of the $p=(n-m+1)$ scalar variables $\left\{\mu_{1}, \mu_{2}, \ldots, \mu_{p}\right\}$ subjected to the constraint $\sum_{k=1}^{p} \mu_{k}=1$ introduced in [3, 4], (ii) these variables along with the constraint, in fact parameterize the null space of the Jacobian, and (iii) self motion inducing joint velocities cannot be obtained by the $B \& C$ solution when the end effector is stationary. The IRC method can induce joint self motion in this configuration. The problem of linearly transforming between the IRC null space parameterizing vector variable and the vector $\left[\mu_{1}, \mu_{2}, \ldots, \mu_{p}\right]^{T}$ is studied. A new constraint free representation of the $\mathrm{B} \& \mathrm{C}$ joint velocity solution is derived and shown to possess the limitation described in (iii).

The FSP method yields a joint velocity solution which induces joint self motion but does not induce end effector motion. The FSP solution is a linear function of $p$ variables $\left\{\omega_{1}, \omega_{2}, \ldots, \omega_{p}\right\}$ which parameterize the null space provided that they satisfy the constraint $\sum_{k=1}^{p} \omega_{k}=0$. By expressing the solution in terms of the IRC basis, it is shown that self motion inducing joint velocities cannot be obtained by the FSP method when the end effector is stationary. Indeed, all joint velocities in the FSP solution are identically equal to zero in this configuration. A new constraint free representation of the FSP joint velocity solution is derived and it too is restricted by this limitation. The linear relationship between the IRC null space parameterizing vector variable and the vector $\left[\omega_{1}, \omega_{2}, \ldots, \omega_{p}\right]^{T}$ is studied. 
Finally, we suggest a unification of the B\&C and FSP null space parameterizations and solution methods. The unification demonstrates that if the designer chooses to parameterize the null space of $J$ using $\left\{\mu_{1}, \mu_{2}, \ldots, \mu_{p}\right\}$ in conjunction with the constraint $\sum_{k=1}^{p} \mu_{k}=1$, then introducing the FSP null space parameters $\left\{\omega_{1}, \omega_{2}, \ldots, \omega_{p}\right\}$ along with the constraint $\sum_{k=1}^{p} \omega_{k}=0$ provide no new information. It is further observed that the unified solution cannot induce joint self motion when the end effector is stationary.

The comparison presented here is based on how the three methods choose variables to parameterize the null space of the Jacobian matrix and on the joint velocity solutions which are explicit functions of these variables. The application of the methods to local optimization is not addressed in this report. It should be mentioned that the comparison of the three redundancy resolution methods is accomplished with the quantities $\{m, n\}$ being general where $(m<n)$.

The report is organized as follows: The three methods are reviewed in section 2. The comparison of the IRC and $B \& C$ methods is presented in section 3 . The IRC and FSP methods are compared in section 4 . The unification of the B\&C and FSP null space parameterizations and joint velocity solution methods is discussed in section 5 . Constraint free representations of the $B \& C$ and FSP joint velocity solutions are derived in terms of the IRC basis and compared to IRC in Appendix A. A summary and conclusion are provided in the final section. 


\section{Review of the Three Methods}

The three redundancy resolution methods developed at ORNL are reviewed in this section.

\subsection{Input Relegation Control}

In Input Relegation Control (IRC) [1], a new vector variable $\epsilon=\left[\epsilon_{1}, \epsilon_{2}, \ldots, \epsilon_{n-m}\right]^{T}$ is introduced which parameterizes the null space of the full rank Jacobian $J$. The vector $\epsilon$ is defined by:

$$
\epsilon=B \dot{q}
$$

where the $n-m$ row vectors comprising matrix $B=B(q)$ are selected such that the composite matrix $S$, defined by:

$$
S=\left[\begin{array}{l}
J \\
B
\end{array}\right]
$$

is nonsingular. It is convenient to partition the inverse of $S$ into two matrices:

$$
S^{-1}=\left[\begin{array}{ll}
E, & F
\end{array}\right]
$$

where $E=E(q)$ and $F=F(q)$ are $(n \times m)$ and $(n \times(n-m))$ matrices, respectively. Eqs. (3) and (4) imply five matrix identities:

$$
J E=I_{m} ; J F=0_{m \times(n-m)} ; B E=0_{(n-m) \times m} ; B F=I_{n-m} ; E J+F B=I_{n} .
$$

Here $I_{k}$ signifies a $(k \times k)$ identity matrix and $0_{k \times l}$ a $(k \times l)$ matrix of zeros. The identity $J F=0_{m \times(n-m)}$ reveals that the column vectors comprising $F$ lie in and span the null space of $J$. It is assumed that the quantities $\{J, F\}$ are known functions of the joint variables $q$ (see $[1,2]$ for methods to determine $F$ given $J$ ).

Eqs. (1) and (2) can be combined and solved for the joint velocities [1]:

$$
\dot{q}=E \dot{x}+F \epsilon .
$$

The first and second terms to the right of eq. (6) are the particular and homogeneous solutions to eq. (1), respectively. The latter term $(F \epsilon)$ induces joint self motions that do not affect end effector motion.

In [2], it was shown that any vector in the $n$-dimensional articular space can be expressed in terms of a basis $V$ defined by:

$$
V=\left[J^{T}, \quad F\right] \text {. }
$$

Indeed, matrix $E$ can be expressed as:

$$
E=J^{T} \beta+F \gamma
$$


where $\beta$ and $\gamma$ are $(m \times m)$ and $((n-m) \times m)$ parameter matrices, respectively. It is easy to verify that $\beta=\left(J J^{T}\right)^{-1}$ and $\gamma=-B J^{T}\left(J J^{T}\right)^{-1}$ by premultiplying eq. (8) by $J$ and $B$, respectively, and noting eq. (5). Substituting the solutions for $\{\beta, \gamma\}$ into eq. (8) yields:

$$
E=J^{T}\left(J J^{T}\right)^{-1}-F B J^{T}\left(J J^{T}\right)^{-1}
$$

Eq. (9) reveals that $(E \dot{x})$ always contains a component $\left(J^{T}\left(J J^{T}\right)^{-1} \dot{x}\right)$ which contributes to the end effector's motion, but it may also contain a component $\left(-F B J^{T}\left(J J^{T}\right)^{-1} \dot{x}\right)$ which induces joint self motion in the general case. If $B$ is selected such that $B^{T}$ lies in the null space of $J$, i.e., $J B^{T}=0_{m \times(n-m)}$, then $(E \dot{x})$ has no self motion component [2].

It is insightful to substitute for $E$ in eq. (6) using eq. (9):

$$
\dot{q}=J^{T}\left(J J^{T}\right)^{-1} \dot{x}-F\left(B J^{T}\left(J J^{T}\right)^{-1} \dot{x}-\epsilon\right) .
$$

Eq. (10) describes a family of all possible solutions to eq. (1) in terms of the basis $V$. (Each solution in the family is distinguished by the designer's choice for the quantities $\{F, B, \epsilon\}$ given $\{J, \dot{x}\}$.) Interestingly, each and every distinct solution in the family has the identical end effector motion inducing component. Therefore, the difference between any two distinct solutions lies in the null space of $J$.

\subsection{Belmans-Culioli Method}

Building upon the combinatorial approach to the inverse kinematics problem proposed in [8], Belmans and Culioli (B\&C) [3,4] suggested a similar technique based on the linear properties of the coordinate transformation in the neighborhood of the point $q$ expressed by the full rank Jacobian $J$ and its $r=C_{m}^{n}$ submatrices $J_{1}, J_{2}, \ldots, J_{k}, \ldots, J_{r}$ of dimension $(m \times m)$. If each $J_{k}$ is assumed invertible, it is possible to obtain $m$-dimensional vectors $\dot{q}_{k}$ such that:

$$
\dot{q}_{k}=J_{k}^{-1} \dot{x},\left(\dot{q}_{k} \in \Re^{m}, k \in\{1,2, \ldots, r\}\right) .
$$

$\mathrm{B} \& \mathrm{C}$ then redefined the vectors $\dot{q}_{k}$ as $n$-dimensional vectors in the articular space, setting the $n-m$ complimentary components to zero. Specifically, if the submatrix $J_{k}$ is formed by removing columns $i_{1}, i_{2}, \ldots, i_{n-m}$ in $J$, then the components of positions $i_{1}, i_{2}, \ldots, i_{n-m}$ are set to zero in the corresponding $n$-dimensional vector $\dot{q}_{k}$. B\&C proposed a solution to eq. (1) which consists of linear combinations of the maximal set of independent vectors $\dot{q}_{k}$, $k=1,2, \ldots, p$ :

$$
\dot{q}\left(\mu_{1}, \mu_{2}, \ldots, \mu_{p}\right)=\mu_{1} \dot{q}_{1}+\mu_{2} \dot{q}_{2}+\ldots+\mu_{p} \dot{q}_{p}
$$

where the scalar variables $\left\{\mu_{1}, \mu_{2}, \ldots, \mu_{p}\right\}$ must be selected to satisfy:

$$
\sum_{k=1}^{p} \mu_{k}=1 .
$$

In eqs. (12) and (13), $p \leq n-m+1$. According to [3, 4]: 
- If $p<n-m+1$, the optimal least norm solution to eq. (1) may not necessarily be obtained using eq. (12) in conjunction with eq. (13).

- If $p=n-m+1$, then the least norm solution is necessarily obtained using eqs. (12) and (13).

In this report it will be assumed that $p=n-m+1$. When using the $\mathrm{B} \& \mathrm{C}$ method, the designer must choose $p$ square and nonsingular submatrices of the Jacobian. The choice is not unique and is, in fact, configuration dependent.

\subsection{Full Space Parameterization Method}

The Full Space Parameterization (FSP) method builds on the earlier work of B\&C [3,4] by proposing a solution to the homogeneous system:

$$
0_{m \times 1}=J \dot{q}
$$

where, here again, it is assumed that $J$ has full rank $m$. Any solution to eq. (14) must lie in the null space of $J$ and will induce self motion of the joints. The solution proposed in [5] is given by:

$$
\dot{q}\left(\omega_{1}, \omega_{2}, \ldots, \omega_{p}\right)=\omega_{1} \dot{q}_{1}+\omega_{2} \dot{q}_{2}+\ldots+\omega_{p} \dot{q}_{p} .
$$

In eq. (15), $\dot{q}_{k}(k \in 1,2, \ldots, p)$ is the $n$-dimensional vector introduced by $\mathrm{B} \& \mathrm{C}$ following eq. (11). The scalar variables $\left\{\omega_{1}, \omega_{2}, \ldots, \omega_{p}\right\}$ in eq. (15) parameterize the null space of $J$ provided that they satisfy:

$$
\sum_{k=1}^{p} \omega_{k}=0
$$

and where, here again, it is assumed that $p=n-m+1$. 


\section{Comparison of B\&C and IRC Methods}

To compare the $\mathrm{B} \& \mathrm{C}$ and IRC methods, it is useful to express the $n$-dimensional vector $\dot{q}_{k}$ defined below eq. (11) in a matrix-column vector form:

$$
\dot{q}_{k}=E_{k} \dot{x}
$$

where the $(n \times m)$ matrix $E_{k}=E_{k}(q)$ has the property that $m$ of its rows are comprised of the rows of the $(m \times m)$ matrix $J_{k}^{-1}$ that was defined in eq. (11). The elements of the $(n-m)$ remaining rows of $E_{k}$ are all equal to zero. It should be noted that eq. (17) is not a function of any variables which parameterize the null space of $J$. Indeed, eq. (17) is a particular solution to eq. (1) where $\dot{q}=\dot{q}_{k}$ and $J E_{k}=I_{m}$.

Suppose that $E_{k}$ is expressed in terms of the basis $V$ defined in eq. (7):

$$
E_{k}=J^{T} \alpha+F \zeta
$$

where $\alpha$ and $\zeta$ are $(m \times m)$ and $((n-m) \times m)$ parameter matrices, respectively. It is easy to verify that $\alpha=\left(J J^{T}\right)^{-1}$ by premultiplying eq. (18) by $J$ and invoking $J F=0_{m \times(n-m)}$. Therefore each of the column vectors comprising $E_{k}$ has a nonzero component lying in the exact range space of $J^{T}$ and the composite matrix $\left[E_{k}, F\right]$ is nonsingular. It is convenient to partition the inverse of $\left[E_{k}, F\right]$ into two matrices:

$$
\left[\begin{array}{ll}
E_{k}, & F
\end{array}\right]^{-1}=\left[\begin{array}{c}
J \\
B_{k}
\end{array}\right]
$$

where the $(n-m) \times n$ matrix $B_{k}$ is the solution for $B$ when $\left\{J, F, E=E_{k}\right\}$ are given. $B_{k}$ relates $\dot{q}_{k}$ and $\epsilon_{k}$ :

$$
\epsilon_{k}=B_{k} \dot{q}_{k}
$$

where the $((n-m) \times 1)$ vector $\epsilon_{k}$ parameterizes the redundant DOF. Substituting for $\dot{q}_{k}$ in eq. (20) using eq. (17) gives:

$$
\epsilon_{k}=B_{k} E_{k} \dot{x}=0_{(n-m) \times 1}
$$

where the matrix identity $B_{k} E_{k}=0_{(n-m) \times m}$ arising from eq. (19) has been applied.

Now, premultiplying eq. (18) by $B_{k}$ gives $\zeta=-B_{k} J^{T}\left(J J^{T}\right)^{-1}$. Substituting for $E_{k}$ in eq. (17) using eq. (18) and applying the solutions for $\{\alpha, \zeta\}$ obtain:

$$
\dot{q}_{k}=J^{T}\left(J J^{T}\right)^{-1} \dot{x}-F B_{k} J^{T}\left(J J^{T}\right)^{-1} \dot{x} .
$$

From another perspective, eq. (17) is just a special case of eq. (6) with $E=E_{k}$, $\epsilon=\epsilon_{k}=0_{(n-m) \times 1}$, and $\dot{q}=\dot{q}_{k}$. Then, applying eq. (10) with $B=B_{k}$ and $\epsilon=\epsilon_{k}$ yields eq. (22).

It is observed that the end effector motion inducing components are identical in the $p(=n-m+1)$ distinct solutions $\left\{\dot{q}_{1}, \dot{q}_{2}, \ldots, \dot{q}_{p}\right\}$ obtained when applying eq. (22). Therefore the difference between two such solutions lies in the null space of $J$.

It is insightful to express the family of particular solutions (to eq. (1)) proposed by $\mathrm{B} \& \mathrm{C}$ in eq. (12) in terms of the basis $V$ : 


$$
\begin{aligned}
\dot{q}\left(\mu_{1}, \mu_{2} \ldots, \mu_{p}\right) & =\sum_{k=1}^{p} \mu_{k} \dot{q}_{k} \\
& =\sum_{k=1}^{p} \mu_{k} J^{T}\left(J J^{T}\right)^{-1} \dot{x}-F\left[\sum_{k=1}^{p} \mu_{k} B_{k}\right] J^{T}\left(J J^{T}\right)^{-1} \dot{x} \\
& =J^{T}\left(J J^{T}\right)^{-1} \dot{x}-F\left[\sum_{k=1}^{p} \mu_{k} B_{k}\right] J^{T}\left(J J^{T}\right)^{-1} \dot{x}
\end{aligned}
$$

where eqs. (13) and (22) have been used. Eq. (23) along with eq. (13) describe the joint space motion of the manipulator.

Eq. (23) reveals that the end effector motion inducing component of the B\&C solution is not a function of the variables $\left\{\mu_{1}, \mu_{2}, \ldots, \mu_{p}\right\}$. Indeed, these variables only affect the self motion of the manipulator and, in fact parameterize the null space of the Jacobian provided that they satisfy eq. (13). These findings are new results not understood in the previous work $[3,4]$.

It will be useful in the ensuing development to express eqs. (13) and (23) in matrixcolumn vector forms:

$$
\begin{gathered}
b^{T} \mu=1, \\
\dot{q}(\mu)=J^{T}\left(J J^{T}\right)^{-1} \dot{x}-F A \mu
\end{gathered}
$$

where $b$ is a $p$-dimensional vector $[1,1, \ldots, 1]^{T}$ and $A=A(q, \dot{x})$ is a $((p-1) \times p)$ matrix in eqs. (24) and (25), respectively. $A$ and the $(p \times 1)$ vector $\mu$ in eqs. (24) and (25) are defined by:

$$
\begin{gathered}
\mu=\left[\begin{array}{lll}
\mu_{1}, & \mu_{2}, \ldots, \mu_{p}
\end{array}\right]^{T}, \\
A=\left[B_{1} J^{T}\left(J J^{T}\right)^{-1} \dot{x}, \quad B_{2} J^{T}\left(J J^{T}\right)^{-1} \dot{x}, \quad \ldots, \quad B_{p} J^{T}\left(J J^{T}\right)^{-1} \dot{x}\right] .
\end{gathered}
$$

We identify a limitation associated with the $\mathrm{B} \& \mathrm{C}$ method and investigate the linear relationship between $\epsilon$ and $\mu$ in the following subsections.

\subsection{Limitation of the B\&C Method}

It is well known that a kinematically redundant manipulator can exhibit joint space motions while the end effector is stationary. Eq. (1) models this configuration when $\dot{x}=0_{m \times 1}$. This choice for $\dot{x}$ results in matrix $A$ in eq. (27) simplifying to:

$$
A=0_{(p-1) \times p} \text {. }
$$

Therefore both terms to the right of eq. (25) vanish when $\dot{x}=0_{m \times 1}$ :

$$
\dot{q}(\mu)=0_{n \times 1}
$$


regardless of the values of the elements of $\mu$. This reveals a limitation with the $\mathrm{B} \& \mathrm{C}$ method. Self motion inducing joint velocities cannot be calculated using eq. (25) when the end effector is stationary. Indeed, eq. (17) simplifies to $\dot{q}_{k}=0_{n \times 1}$ in this configuration, and thus $\dot{q}=\sum_{k=1}^{p} \mu_{k} \dot{q}_{k}$ forms linear combinations of $p$ zero vectors.

On the other hand, the IRC method does not possess this limitation. Indeed, substituting $\dot{x}=0_{m \times 1}$ into eq. (10) yields:

$$
\dot{q}=F \epsilon .
$$

Since $F$ has full rank and the elements of $\epsilon$ are independent, eq. (30) describes all possible solutions for the joint velocities when the end effector is stationary.

\subsection{Linear Relationship Between $\epsilon$ and $\mu$}

The IRC joint velocity solution and the solution obtained using the $\mathrm{B} \& \mathrm{C}$ method are identical when the coefficients of $F$ in eqs. (10) and (25) equate. This leads to a linear relationship between $\epsilon$ and $\mu$ :

$$
\epsilon=B J^{T}\left(J J^{T}\right)^{-1} \dot{x}-A \mu .
$$

Provided that vector $\mu$ is selected such that eq. (24) is satisfied, $\epsilon$ can be calculated as a linear function of $\mu$ using eq. (31).

It is of interest to investigate the inverse relationship, i.e., computing $\mu$ as a linear function of $\epsilon$. To study this problem, it is convenient to combine eqs. (24) and (31) into a single vector equation:

$$
\left[\begin{array}{c}
-1 \\
\epsilon
\end{array}\right]-\left[\begin{array}{c}
0 \\
B J^{T}\left(J J^{T}\right)^{-1} \dot{x}
\end{array}\right]=-C \mu
$$

where the $(p \times p)$ composite matrix $C$ is defined by:

$$
C=\left[\begin{array}{l}
b^{T} \\
A
\end{array}\right]
$$

Given arbitrary values for the elements of $\epsilon$, eq. (32) contains $p$ equations in $p$ unknowns $\mu$. However, when $\dot{x}=0_{m \times 1}, A$ simplifies to eq. (28) and the rank of $C$ reduces to one. Provided that $\epsilon \neq 0_{(n-m) \times 1}$, the equations comprising eq. (32) are inconsistent and cannot be solved for $\mu$ when the end effector is stationary. This confirms that self motion inducing joint velocities calculated using eq. (10) cannot be calculated using eq. (25) when the end effector is stationary.

A constraint free representation of the $B \& C$ solution to eq. (1) is derived and compared to the IRC solution in Appendix A. 


\section{Comparison of FSP and IRC Methods}

It is insightful to express the family of solutions to eq. (14) proposed by FSP in eq. (15) in terms of the basis $V$ defined in eq. (7):

$$
\begin{aligned}
\dot{q}\left(\omega_{1}, \omega_{2} \ldots, \omega_{p}\right) & =\sum_{k=1}^{p} \omega_{k} \dot{q}_{k} \\
& =\sum_{k=1}^{p} \omega_{k} J^{T}\left(J J^{T}\right)^{-1} \dot{x}-F\left[\sum_{k=1}^{p} \omega_{k} B_{k}\right] J^{T}\left(J J^{T}\right)^{-1} \dot{x} \\
& =-F\left[\sum_{k=1}^{p} \omega_{k} B_{k}\right] J^{T}\left(J J^{T}\right)^{-1} \dot{x}
\end{aligned}
$$

where eqs. (16) and (22) have been applied. Eq. (34) confirms that there is no end effector motion inducing component in the FSP joint velocity solution. Eq. (34) along with eq. (16) describe the joint self motions of the manipulator.

It will be useful to express eqs. (16) and (34) in matrix-column vector forms:

$$
\begin{gathered}
b^{T} \omega=0, \\
\dot{q}(\omega)=-F A \omega
\end{gathered}
$$

where $A$ is defined in eq. (27) and $b$ is defined in conjunction with eq. (24). The $(p \times 1)$ vector $\omega$ in eqs. (35) and (36) is defined by:

$$
\omega=\left[\omega_{1}, \omega_{2}, \ldots, \omega_{p}\right]^{T} .
$$

We identify a limitation associated with the FSP method and investigate the linear relationship between $\epsilon$ and $\omega$ in the following subsections.

\subsection{Limitation of the FSP Method}

The FSP method possesses a limitation when the end effector is stationary. When $\dot{x}=$ $0_{m \times 1}, A$ is defined by eq. (28) and eq. (36) simplifies to:

$$
\dot{q}(\omega)=0_{n \times 1}
$$

regardless of the values of the elements of $\omega$. Self motion inducing joint velocity solutions to eq. (14) cannot be obtained by the FSP method.when the end effector is stationary. Indeed, since $\dot{q}_{k}=0_{n \times 1}$ in this configuration, $\dot{q}=\sum_{k=1}^{p} \omega_{k} \dot{q}_{k}$ forms linear combinations of $p$ zero vectors. Furthermore, it is somewhat contradictory to propose a solution to eq. (14) as an explicit function of $\dot{x}$, when eq. (14) is just a special case of eq. (1) with $\dot{x}=0_{m \times 1}$. 


\subsection{Linear Relationship Between $\epsilon$ and $\omega$}

The self motion component of the IRC solution to eq. (1) and the joint velocity solution to eq. (14) obtained by the FSP method are identical when the coefficients of $F$ in eqs. (10) and (36) equate. This leads to a linear relationship between $\epsilon$ and $\omega$ :

$$
\epsilon=B J^{T}\left(J J^{T}\right)^{-1} \dot{x}-A \omega .
$$

Provided that vector $\omega$ is selected such that eq. (35) is satisfied, $\epsilon$ can be calculated as a linear function of $\omega$ using eq. (39).

It is of interest to investigate the inverse relationship, i.e., computing $\omega$ as a linear function of $\epsilon$. To study this problem, it is convenient to combine eqs. (35) and (39) into a single vector equation:

$$
\left[\begin{array}{l}
0 \\
\epsilon
\end{array}\right]-\left[\begin{array}{c}
0 \\
B J^{T}\left(J J^{T}\right)^{-1} \dot{x}
\end{array}\right]=-C \omega
$$

where $C$ is defined in eq. (33). Given arbitrary values for the elements of $\epsilon$, eq. (40) contains $p$ equations in $p$ unknowns $\omega$. However, when $\dot{x}=0_{m \times 1}, C$ is singular with rank one. Provided that $\epsilon \neq 0_{(n-m) \times 1}$, the equations comprising eq. (40) are inconsistent and cannot be solved for $\omega$ when the end effector is stationary. This confirms that self motion inducing joint velocities calculated using eq. (10) cannot be calculated using eq. (36) when the end effector is stationary.

It should be mentioned that the linear relationship between the two sets of dependent null space parameterizations $\mu$ and $\omega$ is established by equating the right sides of eqs. (31) and (39):

$$
A \mu=A \omega
$$

and combining eqs. (24) and (35) into one equation:

$$
b^{T} \mu=1+b^{T} \omega .
$$

Provided that $\dot{x} \neq 0_{m \times 1}$, eqs. (41) and (42) comprise $p$ scalar equations that can be solved to obtain a well specified solution for $\mu(\omega)$ as a function of $\omega(\mu)$. Therefore, if the designer chooses to parameterize the null space of $J$ using vector $\mu$ subject to eq. (24), then the null space parameterization $\omega$ subject to eq. (35) introduced in [5] provides no new information.

A constraint free representation of the FSP solution to eq. (14) is derived and compared to the self motion component of the IRC solution to eq. (1) in Appendix A. 


\section{Unification of B\&C and FSP Null Space Parameterizations and Solution Methods}

We have shown here for the first time that the set of variables $\left\{\mu_{1}, \mu_{2}, \ldots, \mu_{p}\right\}$ introduced in [3] parameterize the null space of $J$ provided that eq. (13) is satisfied. The purpose of this section is to propose a unification of this parameterization and the one suggested in [5], which leads to a unification of the B\&C and FSP joint velocity solution methods. It will be shown that the unified result possesses the same limitation associated with the individual methods.

We propose a new set of variables $\left\{\nu_{1}, \nu_{2}, \ldots, \nu_{p}\right\}$, where $\nu_{k}$ is defined by:

$$
\nu_{k}=\mu_{k}+a \omega_{k} ; \quad k \in\{1,2, \ldots, p\}
$$

where $a$ is an arbitrary constant. Noting eqs. (13) and (16), it is easy to verify that:

$$
\sum_{k=1}^{p} \nu_{k}=\sum_{k=1}^{p} \mu_{k}+a \sum_{k=1}^{p} \omega_{k}=1 .
$$

A family of particular solutions to eq. (1) can be expressed in terms of the variables $\left\{\nu_{1}, \ldots, \nu_{p}\right\}$ :

$$
\dot{q}\left(\nu_{1}, \nu_{2} \ldots, \nu_{p}\right)=\sum_{k=1}^{p} \nu_{k} \dot{q}_{k}
$$

where $\dot{q}_{k}$ is defined below eq. (11). Substituting for $\dot{q}_{k}$ in eq. (45) using eq. (22) expresses the joint velocity solution in terms of the basis $V$ :

$$
\begin{aligned}
\dot{q}\left(\nu_{1}, \nu_{2} \ldots, \nu_{p}\right) & =\sum_{k=1}^{p} \nu_{k} J^{T}\left(J J^{T}\right)^{-1} \dot{x}-F\left[\sum_{k=1}^{p} \nu_{k} B_{k}\right] J^{T}\left(J J^{T}\right)^{-1} \dot{x} \\
& =J^{T}\left(J J^{T}\right)^{-1} \dot{x}-F\left[\sum_{k=1}^{p} \nu_{k} B_{k}\right] J^{T}\left(J J^{T}\right)^{-1} \dot{x}
\end{aligned}
$$

Eqs. (44) and (46) reveal that the set of variables $\left\{\nu_{1}, \nu_{2}, \ldots, \nu_{p}\right\}$ constitutes a family of null space parameterizations of the Jacobian.

The joint velocity solution in eq. (46) reveals that if we choose to parameterize the null space of $J$ using $\left\{\mu_{1}, \mu_{2}, \ldots, \mu_{p}\right\}$ subject to eq. (13), then the null space parameterization $\left\{\omega_{1}, \omega_{2}, \ldots, \omega_{p}\right\}$ subject to eq. (16) introduced in [5] provides no new information.

We further observe that the joint velocity solution in eq. (46) yields $\dot{q}=0_{n \times 1}$ when $\dot{x}=0_{m \times 1}$. 


\section{Discussion and Conclusion}

The report has provided an analytical comparison of three redundancy resolution methods developed by researchers at the Oak Ridge National Laboratory. We have focused on how the methods choose variables to parameterize the null space of the full rank $(m \times n)$ Jacobian matrix $J$ and on the joint velocity solutions which are explicit functions of these variables. The Input Relegation Control (IRC) method describes all possible solutions to the kinematic velocity model (eq. (1)) using linear combinations of two orthogonal subspaces: the $m$ dimensional exact range space of the transposed Jacobian and the $(n-m)$-dimensional null space (of the Jacobian). The null space is parameterized using the minimal number of variables $\epsilon=\left[\epsilon_{1}, \epsilon_{2}, \ldots, \epsilon_{n-m}\right]^{T}$. The components of $\epsilon$ are independent.

The joint velocity solution obtained by IRC (see eq. (6)) consists of a particular solution $(E \dot{x})$ and a homogeneous solution $(F \epsilon)$, where the $(n-m)$ column vectors comprising matrix $F$ span the null space of $J$. By expressing matrix $E$ in terms of the basis $\left[J^{T}, F\right]$, it was shown that the particular solution consists of an end effector motion inducing component $\left\{J^{T}\left(J J^{T}\right)^{-1} \dot{x}\right\}$ and a joint self motion inducing component $\left\{-F B J^{T}\left(J J^{T}\right)^{-1} \dot{x}\right\}$ (see eq. (10)). The homogeneous component $(F \epsilon)$ also induces joint self motion.

After reviewing the other two methods, the comparison commenced by expressing the Belmans and Culioli (B\&C) solution shown in eq. (12) in terms of the basis $\left[J^{T}, F\right]$ (see eqs. (23) and (25)). This separated it into its end effector motion and self motion inducing components, where each component is a linear function of the end effector Cartesian velocity vector $\dot{x}$. Indeed, it was shown that eq. (12) is just a linear combination of particular solutions to eq. (1). Furthermore, expressing the B\&C solution in terms of $\left[J^{T}, F\right]$ led to three new insightful observations about the B\&C solution: (i) only the self motion component is a function of the $p(=n-m+1)$ scalar variables $\left\{\mu_{1}, \mu_{2}, \ldots, \mu_{p}\right\}$ subjected to the constraint $\sum_{k=1}^{p} \mu_{k}=1$ introduced in [3,4], (ii) these variables along with the constraint, in fact parameterize the null space of the Jacobian, and (iii) self motion inducing joint velocities cannot be obtained by the $\mathrm{B} \& \mathrm{C}$ solution when the end effector is stationary. Indeed, when $\dot{x}=0_{m \times 1}$, eq. (25) reduces to $\dot{q}=0_{n \times 1}$. The IRC solution can induce joint self motion when the end effector is stationary.

A linear relationship between $\epsilon$ and $\mu$ was investigated. The results indicated that $\epsilon$ can be calculated as a linear function of $\mu$, provided that the components of $\mu$ satisfy the constraint $\sum_{k=1}^{p} \mu_{k}=1$. However, it was discovered that $\mu$ cannot be calculated as a linear function of $\epsilon$ when the end effector is stationary and at least one component of $\epsilon$ is nonzero. Therefore any joint velocity solution obtained by the $\mathrm{B} \& \mathrm{C}$ method can be calculated by the IRC method, but non-zero joint velocity solutions obtained by IRC when $\dot{x}=0_{m \times 1}$ cannot be calculated by the $\mathrm{B} \& \mathrm{C}$ method.

A constraint free representation of the $\mathrm{B} \& \mathrm{C}$ solution was obtained by solving for $\mu_{p}$ as a function of $\left\{\mu_{1}, \mu_{2}, \ldots, \mu_{p-1}\right\}$ based on the constraint $\sum_{k=1}^{p} \mu_{k}=1$ and substituting the result into eq. (23). It was shown that all joint velocities in the resulting solution given by eq. (A.3) are identically equal to zero regardless of the value of null space parameterizing vector $\mu^{s}\left(=\left[\mu_{1}, \mu_{2}, \ldots, \mu_{p-1}\right]^{T}\right)$ when the end effector is stationary. It was shown that $\epsilon$ can be expressed as a linear function of $\mu^{s}$, but $\mu^{s}$ cannot be calculated as a linear function of $\epsilon$ when $\dot{x}=0_{m \times 1}$ and at least one element of $\epsilon$ is nonzero.

The Full Space Parameterization (FSP) method proposed a joint velocity solution which induces joint self motion by solving the homogeneous system $0_{m \times 1}=J \dot{q}$. The solution 
proposed by FSP (see eq. (15)) is a function of the variables $\left\{\omega_{1}, \omega_{2}, \ldots, \omega_{p}\right\}$, which parameterize the null space of $J$ provided that the constraint $\sum_{k=1}^{p} \omega_{k}=0$ is satisfied. Eq. (15) was expressed in terms of the basis $\left[J^{T}, F\right]$ (see eqs. (34) and (36)), which revealed that all joint velocities in the FSP solution are identically equal to zero at $\dot{x}=0_{m \times 1}$, regardless of the values of $\left\{\omega_{1}, \omega_{2}, \ldots, \omega_{p}\right\}$. Therefore the method cannot induce joint self motion when the end effector is stationary.

A linear relationship between $\epsilon$ and $\omega\left(=\left[\omega_{1}, \omega_{2}, \ldots, \omega_{p}\right]^{T}\right)$ was established and it was shown that $\epsilon$ can be calculated as a function of $\omega$, provided that the values of the elements of $\omega$ satisfy $\sum_{k=1}^{p} \omega_{k}=0$. Therefore any joint velocity solution obtained by the FSP method can be calculated using the self motion component of the IRC solution. However, $\omega$ cannot be expressed as a linear function of $\epsilon$ when $\dot{x}=0_{m \times 1}$ and at least one element of $\epsilon$ is nonzero.

A constraint free representation of the FSP solution to $0_{m \times 1}=J \dot{q}$ was obtained by solving for $\omega_{p}$ as a function of $\left\{\omega_{1}, \omega_{2}, \ldots, \omega_{p-1}\right\}$ based on the constraint $\sum_{k=1}^{p} \omega_{k}=0$ and substituting the result into eq. (34). The resulting solution given by eq. (A.10) is identically equal to zero when the end effector is stationary, regardless of the values of the elements of $\omega^{s}\left(=\left[\omega_{1}, \omega_{2}, \ldots, \omega_{p-1}\right]^{T}\right)$. It was also shown that $\epsilon$ can be expressed as a linear function of $\omega^{s}$, but $\omega^{s}$ cannot be calculated as a linear function of $\epsilon$ when $\dot{x}=0_{m \times 1}$ and at least one element of $\epsilon$ is nonzero.

In section 5 , we suggested how the null space parameterizations of the $\mathrm{B} \& \mathrm{C}$ and FSP methods can be combined to define a family of null space parameterizations $\left\{\nu_{1}, \nu_{2}, \ldots, \nu_{p}\right\}$, where $\nu_{k}=\mu_{k}+a \omega_{k}$ and $\sum_{k=1}^{p} \nu_{k}=1$. Then, it was shown that $\dot{q}=\sum_{k=1}^{p} \nu_{k} \dot{q}_{k}$ constitutes a family of particular solutions to eq. (1), and that all joint velocities in the solution are identically equal to zero when the end effector is stationary regardless of the values of $\left\{\nu_{1}, \nu_{2}, \ldots, \nu_{p}\right\}$. It was concluded that if the designer chooses to use the set $\left\{\mu_{1}, \mu_{2}, \ldots, \mu_{p}\right\}(p=n-m+1)$ subjected to the constraint $\sum_{k=1}^{p} \mu_{k}=1$ to parameterize the null space, then introducing a different set of variables $\left\{\omega_{1}, \omega_{2}, \ldots, \omega_{p}\right\}$ subjected to $\sum_{k=1}^{p} \omega_{k}=0$ to parameterize the null space provides no new information.

It is logical and reasonable that a kinematic redundancy resolution method should be able to describe the joint self motion of a manipulator when its end effector is moving or is stationary. The IRC method is capable of doing this and provides all possible solutions to eq. (1) using a minimal number of null space parameters. The authors conclude that the IRC method proposed by them is superior to the B\&C [3, 4] and FSP [5] methods. 


\section{Acknowledgement}

This research was sponsored by the Office of Engineering Research Program, Basic Energy Sciences, U.S. Department of Energy, under Contract No. DE-AC05-960R22464 with Lockheed Martin Energy Research Corporation.

The authors wish to thank Dr. Lynne E. Parker for encouraging the continuation and completion of this research. 


\section{References}

[1] M.A. Unseren, "Input Relegation Control for Gross Motion of a Kinematically Redundant Manipulator," Oak Ridge National Laboratory Technical Report No. ORNL/TM12165, October 1992.

[2] M.A. Unseren and D.B. Reister, "New Insights Into Input Relegation Control for Inverse Kinematics of a Redundant Manipulator," Oak Ridge National Laboratory Technical Report No. ORNL/TM-12813 [Part 1], (July 1995) Oak Ridge, TN, USA.

[3] P. Belmans and J.-C. Culioli, "An Alternate Method to Solving the Kinematics of a Redundant Robot," Proceedings of the 1990 Annual Meeting of the American Nuclear Society, Nashville, TN, June 10-14, 1990.

[4] P. Belmans and J.-C. Culioli, "A New Approach to Solve the Kinematics Resolution of a Redundant Robot," Oak Ridge National Laboratory Technical Report No. ORNL/TM11435, (March 1990) Oak Ridge, TN, USA.

[5] F.G. Pin, P. Belmans, J.-C. Culioli, D.D. Carlson, and F.A. Tulloch, "A New Solution Method for the Inverse Kinematic Joint Velocity Calculations of Redundant Manipulators," Proceedings of the 1994 IEEE Int'l Conference on Robotics and Automation, pp. 96-102, San Diego, CA, May 1994.

[6] D.N. Nenchev, "Redundancy Resolution through Local Optimization: A Review," J. of Robotic Systems Vol. 6, No. 6, pp. 769-798, December 1989.

[7] B. Siciliano, "Kinematic Control of Redundant Robot Manipulators: A Tutorial," $J$. of Intelligent and Robotic Systems Vol. 3, pp. 201-212, 1990.

[8] S. Hirose and S. Ma, "Redundancy Decomposition Control for Multi-Joint Manipulator," Proc. IEEE Int'1. Conf. on Robotics and Automation, Scottsdale, AZ, pp. 119-124, May, 1989.

[9] B. Noble and J.W. Daniel, Applied Linear Algebra, 2nd ed., Prentice-Hall, Inc., 1977. 


\section{Appendix A. Comparison of IRC to Constraint Free Representations of B\&C and FSP Solutions}

The designer must choose values for the null space parameters $\left\{\mu_{1}, \mu_{2}, \ldots, \mu_{p}\right\}$ which satisfy eq. (13) when calculating a solution to eq. (1) using eq. (23). Likewise, values for the null space parameters $\left\{\omega_{1}, \omega_{2}, \ldots, \omega_{p}\right\}$ must satisfy eq. (16) when calculating a solution to eq. (14) using eq. (34). The purpose of this Appendix is to express the B\&C and FSP joint velocity solutions in constraint free representations and compare them to the IRC joint velocity solution.

\section{A.1 Constraint Free Representation of B\&C Solution}

We will express eq. (23) in terms of an independent subset of $\left\{\mu_{1}, \mu_{2}, \ldots, \mu_{p}\right\}$. Without loss of generality, $\mu_{p}$ will become an implicit variable. Substituting for $\mu_{p}$ in eq. (23) using:

$$
\mu_{p}=1-\sum_{k=1}^{p-1} \mu_{k}
$$

and rearranging terms yield:

$\dot{q}\left(\mu_{1}, \mu_{2}, \ldots, \mu_{p-1}\right)=J^{T}\left(J J^{T}\right)^{-1} \dot{x}-F\left\{\left[\sum_{k=1}^{p-1} \mu_{k}\left(B_{k}-B_{p}\right)\right]+B_{p}\right\} J^{T}\left(J J^{T}\right)^{-1} \dot{x}$

where, here again, $p=n-m+1$. The designer may now calculate the joint velocity solution to eq. (1) obtained by the $B \& C$ method in a constraint free manner using eq. (A.2).

For the ensuing comparison it will be useful to express eq. (A.2) in a matrix-column vector form:

$$
\dot{q}\left(\mu^{s}\right)=\left(I_{n}-F B_{p}\right) J^{T}\left(J J^{T}\right)^{-1} \dot{x}-F D \mu^{s}
$$

where $\mu^{s}$ is a $((p-1) \times 1)$ vector and $D=D(q, \dot{x})$ is a $((p-1) \times(p-1))$ matrix. These quantities are defined by:

$$
\begin{gathered}
\mu^{s}=\left[\begin{array}{lll}
\mu_{1}, & \mu_{2}, & \ldots, \mu_{p-1}
\end{array}\right]^{T} \\
D=\left[\left(B_{1}-B_{p}\right) J^{T}\left(J J^{T}\right)^{-1} \dot{x} \quad, \quad\left(B_{2}-B_{p}\right) J^{T}\left(J J^{T}\right)^{-1} \dot{x}\right. \\
\ldots \\
\left.\ldots,\left(B_{p-1}-B_{p}\right) J^{T}\left(J J^{T}\right)^{-1} \dot{x}\right] .
\end{gathered}
$$

\section{A.1.1 Limitation}

It is easy to see that $D=0_{(p-1) \times(p-1)}$ when $\dot{x}=0_{m \times 1}$. With this choice of $\dot{x}$, eq. (A.3) simplifies to: 


$$
\dot{q}\left(\mu^{s}\right)=0_{n \times I}
$$

regardless of the values of the elements of $\mu^{s}$. This reveals that the constraint free representation of the $\mathrm{B} \& \mathrm{C}$ joint velocity solution to eq. (1) cannot induce joint self motion when the end effector is stationary.

\section{A.1.2 Linear Relationship Between $\epsilon$ and $\mu^{s}$}

The joint velocity solution obtained by IRC and the constraint free representation of the $\mathrm{B} \& \mathrm{C}$ solution are identical when the coefficients of $F$ in eqs. (10) and (A.3) equate. This results in $\epsilon$ being expressed as a linear function of $\mu^{s}$ :

$$
\epsilon=\left(B-B_{p}\right) J^{T}\left(J J^{T}\right)^{-1} \dot{x}-D \mu^{s} .
$$

It is of interest to investigate the inverse relationship, i.e., computing $\mu^{s}$ as a linear function of $\epsilon$. Given arbitrary values for the elements of $\epsilon$, eq. (A.7) contains $p-1$ equations in $p-1$ unknowns $\mu^{s}$. However, when $\dot{x}=0_{m \times 1}$, matrix $D=0_{(p-1) \times(p-1)}$. Provided that $\epsilon \neq 0_{(n-m) \times 1}$, the equations comprising eq. (A.7) are inconsistent and cannot be solved for $\mu^{s}$ when the end effector is stationary.

\section{A.2 Constraint Free Representation of FSP Solution}

We will express eq. (34) in terms of an independent subset of $\left\{\omega_{1}, \omega_{2}, \ldots, \omega_{p}\right\}$ in this section. Without loss of generality, $\omega_{p}$ will be become an implicit variable. Substituting for $\omega_{p}$ in eq. (34) using:

$$
\omega_{p}=-\sum_{k=1}^{p-1} \omega_{k}
$$

and rearranging terms yield:

$$
\dot{q}\left(\omega_{1}, \omega_{2} \ldots, \omega_{p-1}\right)=-F\left[\sum_{k=1}^{p-1} \omega_{k}\left(B_{k}-B_{p}\right)\right] J^{T}\left(J J^{T}\right)^{-1} \dot{x} .
$$

The designer may now calculate the joint velocity solution to eq. (14) obtained by the FSP method in a constraint free manner using eq. (A.9).

It is helpful to express eq. (A.9) in a matrix-column vector form:

$$
\dot{q}\left(\omega^{s}\right)=-F D \omega^{s}
$$

where $D$ is defined in eq. (A.5) and the $((p-1) \times 1)$ vector $\omega^{s}$ is given by:

$$
\omega^{s}=\left[\begin{array}{lll}
\omega_{1}, & \omega_{2}, & \ldots, \omega_{p-1}
\end{array}\right]^{T} .
$$




\section{A.2.1 Limitation}

When $\dot{x}=0_{m \times 1}, D=0_{(p-1) \times(p-1)}$ and thus eq. (A.10) simplifies to:

$$
\dot{q}\left(\omega^{s}\right)=0_{n \times 1}
$$

regardless of the values of the elements of $\omega^{s}$. This reveals that the constraint free representation of the FSP joint velocity solution to eq. (14) cannot induce joint self motion when the end effector is stationary.

\section{A.2.2 Linear Relationship Between $\epsilon$ and $\omega^{s}$}

The self motion component of the solution to eq. (1) obtained by IRC and the constraint free representation of the FSP solution to eq. (14) are identical when the coefficients of $F$ in eqs. (10) and (A.10) equate. This results in $\epsilon$ being expressed as a linear function of $\omega^{s}$ :

$$
\epsilon=B J^{T}\left(J J^{T}\right)^{-1} \dot{x}-D \omega^{s} .
$$

It is of interest to investigate the inverse relationship, i.e., computing $\omega^{s}$ as a linear function of $\epsilon$. Given arbitrary values for the elements of $\epsilon$, eq. (A.13) contains $p-1$ equations in $p-1$ unknowns $\omega^{s}$. However, when $\dot{x}=0_{m \times 1}, D=0_{(p-1) \times(p-1)}$. Provided that $\epsilon \neq 0_{(n-m) \times 1}$, the equations comprising eq. (A.13) are inconsistent and cannot be solved for $\omega^{s}$ when the end effector is stationary. 
ORNL/TM-13510

\section{INTERNAL DISTRIBUTION}

1-5. J. Barhen

6. C. W. Glover

7. W. C. Grimmell

8-12. M. A. Kuliasha

13. E. M. Oblow

14. C. E. Oliver

15-19. L. E. Parker

20. V. Protopopescu

21. N. S. Rao
22-26. D. B. Reister

27. M. S. Smith

28-32. M. A. Unseren

33. CSMD Reports Office

34. Central Research Library

35. Document Reference Section

36. Laboratory Records - RC

37. ORNL Patent Office

\section{EXTERNAL DISTRIBUTION}

38-39. Office of Scientific and Technical Information, P.O. Box 62, Oak Ridge, TN 37831.

40. Dr. Fred Aminzadeh, UNOCAL, 14141 S.W. Freeway, Suite 301-225, Sugarland, TX 77478.

41. Dr. John Baillieul, Aerospace and Mechanical Engineering Department, Boston University, 110 Cummington Street, Boston, MA 02215.

42. Dr. John Blair, JBX Technologies, 25 Moore Road, Wayland, MA 01778.

43. Dr. Roger W. Brockett, Harvard University, Pierce Hall, 29 Oxford Street, Cambridge, MA 02138.

44. Dr. Pasquale Chiacchio, Universita degli Studi di Napoli Federico II, Dipartimento di Informatica e Sistemistica, Via Claudio, 21, 80125 Napoli, ITALY.

45. Dr. Stefano Chiaverini, Universita degli Studi di Napoli Federico II, Dipartimento di Informatica e Sistemistica, Via Claudio, 21, 80125 Napoli, ITALY.

46. Dr. Hooshang Hemami, Department of Electrical Engineering, Ohio State University, 2015 Neil Avenue, Columbus, $\mathrm{OH} 43210$.

47. Professor Ping Hsu, Department of Electrical Engineering, San Jose State University, San Jose, CA 95192.

48. Dr. Oscar P. Manley, 13212 Wye Oak Drive, Gaithersburg, MD 20878.

49. Dr. Robert E. Price, ER-15, Office of Basic Energy Sciences, U.S. Department of Energy, Washington, DC 20585.

50. R. D. Rafler, 8906 Talbot Avenue, Silver Spring, MD 20910.

51. Dr. Rodney G. Roberts, Department of Electrical Engineering, FAMU/FSU College of Engineering, Florida State University, 2525 Pottsdamer Street, Tallahassee, FL 323106046.

52. Dr. Ian D. Walker, Department of Electrical and Computer Engineering, Clemson University, Clemson, SC 29634.

53. Professor Li-Chun T. Wang, Department of Mechanical Engineering, National Taiwan Institute of Technology, 43 Keelung Road, Section 4, Taipei, Taiwan, 106, REPUBLIC OF CHINA. 
54. Dr. John T. Wen, Center for Advanced Technology for Automation, Robotics and Manufacturing, Department of Electrical, Computer, and Systems Engineering, Rensselaer Polytechnic Institute, Troy, NY 12180.

55. Dr. Charles R. Weisbin, Robotics and Mars Exploration Technology Program, Jet Propulsion Laboratory, 4800 Oak Grove Drive, Pasadena, CA 91109.

56. Professor Xiaoping Yun, Electrical and Computer Engineering, Naval Postgraduate School, Code EC/YX, Monterey, CA 93943-5121.

57. Dr. Yong Zhou, Compumotor Division, Parker Hannifin Corporation, 5500 Business Park Drive, Rohnert Park, CA 94928. 